\title{
Acompanhamento do Processamento de Elastômeros Condutores por Microscopia Eletrônica de Varredura
}

\author{
Roselena Faez, Inácio M. Martin, Mirabel C. Rezende \\ Divisão de Materiais, Instituto de Aeronáutica e Espaço, CTA
}

Marco-Aurélio De Paoli Laboratório de Polímeros Condutores e Reciclagem, Instituto de Química, UNICAMP

\begin{abstract}
Resumo: Neste trabalho, o elastômero EPDM foi misturado à Polianilina (PAni) a qual foi dopada com ácido dodecilbenzeno sulfônico (DBSA) na razão molar 1:3. A mistura de EPDM contendo $40 \%(\mathrm{~m} / \mathrm{m})$ de PAni(DBSA) ${ }_{3}$ foi realizada em um misturador interno de dois rotores, acessório do reômetro Haake Rheocord 90, a $150^{\circ} \mathrm{C}$ e $30 \mathrm{rpm}$. Foram recolhidas amostras em 5, 10, 20, 30 e 40 min de processamento e a morfologia foi avaliada por MEV. A análise microscópica da superfície da amostra mostrou fases completamente distintas em função do tempo de mistura, passando de uma estrutura de duas fases compacta (5 min) até o aparecimento de uma estrutura "tipo esponja" (30 e $40 \mathrm{~min}$ ). Estas diferenças afetam as propriedades do material como, por exemplo, o comportamento de absorção de radiação eletromagnética de materiais absorvedores de radiação (MARE).
\end{abstract}

Palavras-chave: Elastômero condutor, polianilina, EPDM, morfologia, MARE.

\section{Evaluation of a Conductive Elastomer Processing Using Scanning Electron Microscopy}

Abstract: In this work the EPDM elastomer was mixed with Polyaniline (PAni) doped with dodecilbenzene sulfonic acid (DBSA) using a molar ratio of 1:3 of PAni:DBSA. The EPDM mixture with $40 \%(\mathrm{w} / \mathrm{w})$ of PAni(DBSA) $)_{3}$ was carried out using an internal mixer chamber with two rotors coupled to a Haake Rheocord 90 at $150^{\circ} \mathrm{C}$ and $30 \mathrm{rpm}$. Aliquots were taken during the processing time of 5, 10, 20, 30 and $40 \mathrm{~min}$, and the morphology was evaluated by SEM. The morphology of the blends markedly changes as a function of processing time, from a compact, at $5 \mathrm{~min}$ to a sponge-like morphology at 30 and $40 \mathrm{~min}$. These differences influence the material properties, such as electromagnetic radiation absorption of radar absorbing materials (RAM).

Keywords: Conductive elastomer, polyaniline, EPDM, morphology, RAM.

\section{Introdução}

Os elastômeros e termoplásticos condutores têm sido extensivamente estudados e utilizados na blindagem de interferência eletromagnética, sensores, dissipação de carga estática, absorvedores de radiação eletromagnética, dentre outros ${ }^{[1,2]}$. Esses vêm sendo preparados, principalmente, pela inclusão de cargas condutoras como negro de fumo condutor, fibra de carbono ou fibras metálicas, e são chamados de elastômeros condutores extrínsecos. Vários exemplos de sua utilização podem ser citados, como: em tele- fones, alguns componentes de automóveis, gabinetes de televisores, etc. O grande inconveniente do uso de polímeros condutores extrínsecos é com relação à massa específica do material final que é alta, associada ao empobrecimento das propriedades mecânicas do polímero isolante utilizado. Nesse contexto está a vantagem em se utilizar sistemas contendo polímeros condutores intrínsecos (PCI), os quais apresentam densidades menores. Nesse caso, faz-se a preparação de blendas de um polímero convencional (também conhecido como isolante) e um PCI. Várias técnicas são utilizadas para a preparação de blendas,

Autor para correspondência: Mirabel C. Rezende, CTA, Instituto de Aeronáutica e Espaço, Divisão de Materiais, Praça Marechal do Ar Eduardo Gomes 50, CEP: 12288-904, São José dos Campos , SP. E-mail: mirabel@iae.cta.br 
como: evaporação de uma solução que contenha os componentes da mistura ${ }^{[3,4]}$, polimerização do polímero condutor em uma matriz, sendo que este pode ser preparado química ${ }^{[5]}$ ou eletroquimicamente ${ }^{[6-8]}$ ou por mistura mecânica usando uma câmara de mistura (tipo Brabender) ou extrusoras ${ }^{[9]}$.

Alguns parâmetros são importantes na preparação de absorvedores de radiação eletromagnética baseados em polímeros intrinsecamente condutores (PIC). Estes possuem uma característica determinante para o seu uso como centro absorvedor que é a variação da condutividade, e portanto, da constante dielétrica em função da freqüência da onda incidente. No entanto, esta variável é influenciada por outros parâmetros como: tamanho da cadeia, o nível de dopagem, o tipo de dopante, o método de síntese do polímero condutor bem como o método de preparação das misturas com polímeros isolantes ${ }^{[10]}$. Assim, é necessário ter um controle bastante rígido dos parâmetros experimentais, principalmente em se tratando de uma mistura é primordial o entendimento do comportamento das fases formadas, pois estas podem modificar as propriedades finais do material.

O objetivo deste trabalho é estudar a morfologia das fases de uma blenda elastomérica condutora, por meio de análises efetuadas por microscopia eletrônica de varredura, a partir de amostras provenientes de diferentes etapas do processamento de mistura mecânica e correlacioná-la com a absorção de radiação eletromagnética.

\section{Experimental}

\section{Síntese da Polianilina}

A PAni foi sintetizada quimicamente em escala pré-piloto (reator de vidro encamisado com capacidade de $10 \mathrm{~L}$ ) usando $\left(\mathrm{NH}_{4}\right)_{2} \mathrm{~S}_{2} \mathrm{O}_{8}$ como oxidante em solução ácida de $\mathrm{HCl}$. O pó obtido foi filtrado, lavado com água destilada até obtenção de filtrado incolor ${ }^{[11]}$. A polianilina dopada com $\mathrm{HCl}$ foi desdopada com uma solução aquosa de $\mathrm{NH}_{4} \mathrm{OH} 1,0 \mathrm{~mol} \mathrm{~L}^{-1} \mathrm{du}-$ rante $24 \mathrm{~h}$ para se obter a PAni base esmeraldina na forma de um pó preto. A dopagem da PAni base esmeraldina em meio DBSA foi realizada por processamento reativo no misturador interno de dois rotores contra-rotatórios, acessório do equipamento reômetro Haake Rheocord $90^{[11,12]}$.

\section{Preparação da blenda}

Para estudar o efeito do processamento na morfologia da blenda EPDM/PAni-(DBSA) ${ }_{3}$ preparou-se uma mistura contendo $40 \%(\mathrm{~m} / \mathrm{m})$ de PAni$(\mathrm{DBSA})_{3}$ no misturador interno do reômetro Haake Rheocord 90 na sequiência descrita a seguir. Foram adicionados simultaneamente o EPDM, a PAni base esmeraldina e o DBSA a $150^{\circ} \mathrm{C}$ e $30 \mathrm{rpm}$. Retirou-se alíquotas a 5, 10, 20, 30 e $40 \mathrm{~min}$. As alíquotas das amostras foram prensadas em uma prensa hidráulica aquecida a $100^{\circ} \mathrm{C}$ por $10 \mathrm{~min}$ sob pressão de $6 \mathrm{MPa}$.

Em seguida, as amostras foram analisadas por microscopia eletrônica de varredura utilizando-se um equipamento Digital Scanning Microscope DSM 950 da Zeiss, operando em $25 \mathrm{kV}$. As análises da superfície da amostra foram feitas sem a necessidade de metalização, pois estas apresentam condutividade suficiente para a sua realização.

\section{Resultados e Discussão}

Por microscopia eletrônica de varredura (MEV) foi realizado um estudo do efeito do tempo de processamento de uma amostra de EPDM/PAni$(\text { DBSA })_{3}(60 / 40)$ para verificar possíveis modificações na sua morfologia. A Figura 1 mostra a curva de torque versus tempo de mistura. As descontinuidades da curva indicam os pontos onde foram recolhidas as alíquotas para a caracterização.

Por meio das curvas de torque em função do tempo de processamento é possível acompanhar as prováveis reações que ocorrem durante a mistura. Estas reações são: (i) dopagem da PAni base pelo ácido

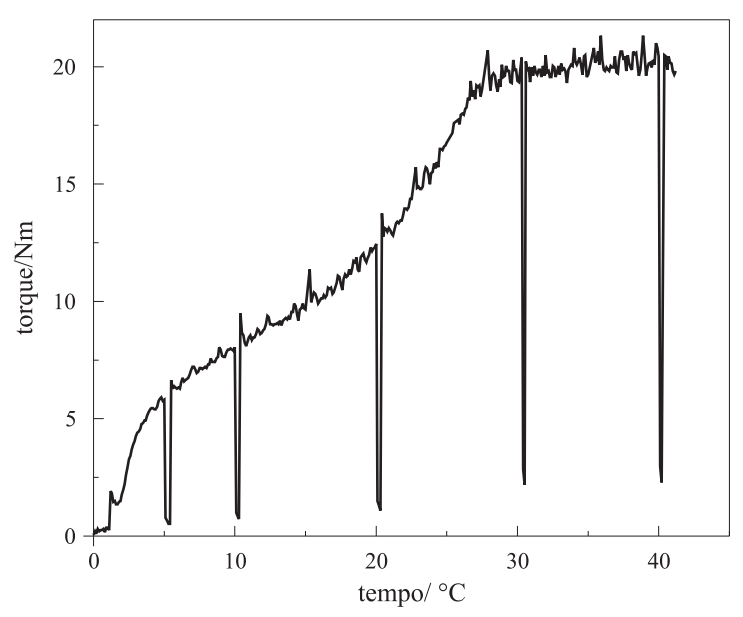

Figura 1. Curva de torque versus tempo de mistura da blenda EPDM/ PAni-(DBSA) 
Faez, R. etal - Processamento de elastômeros condutores por MEV

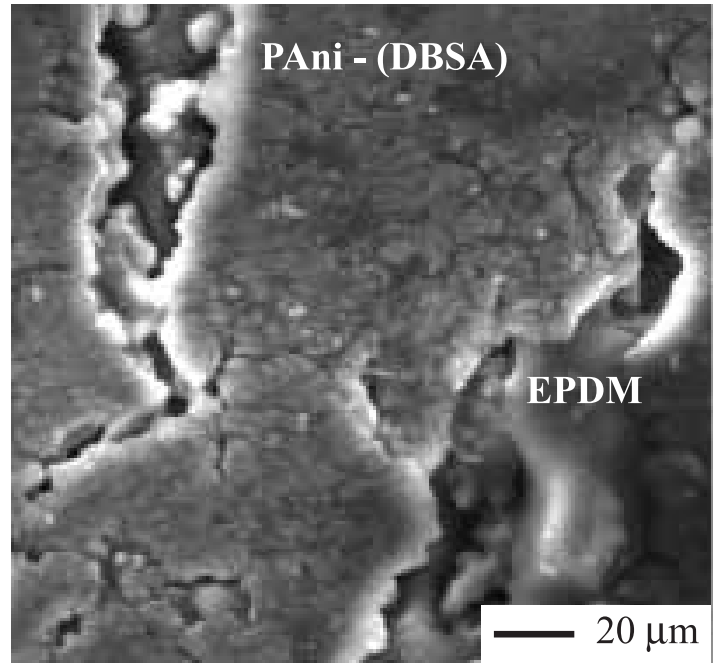

(a)

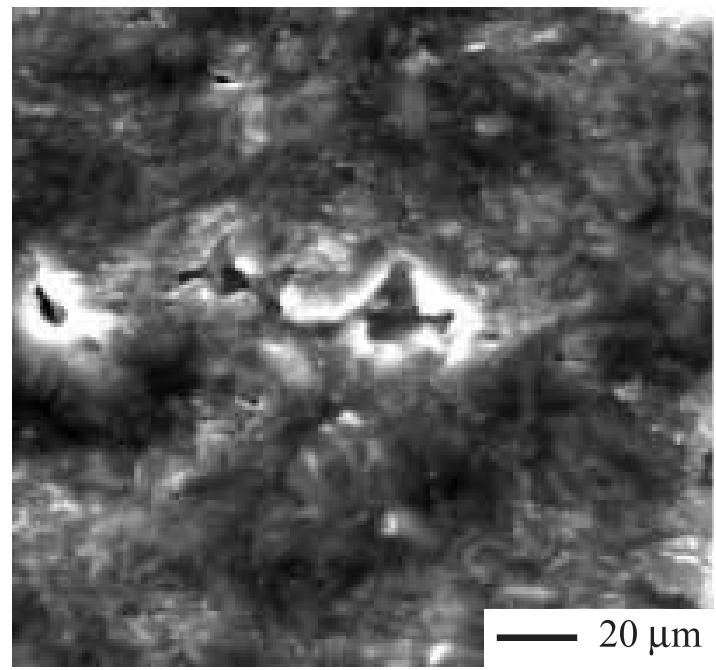

(c)

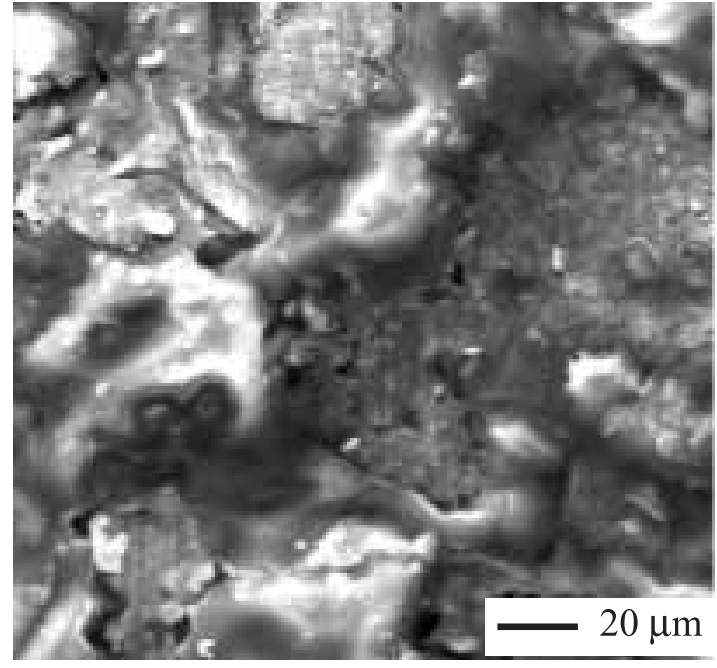

(b)

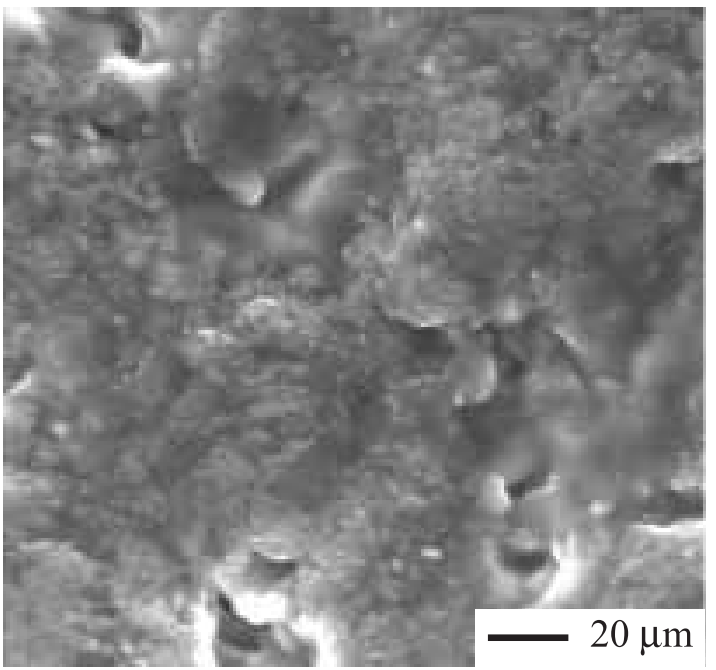

(d)

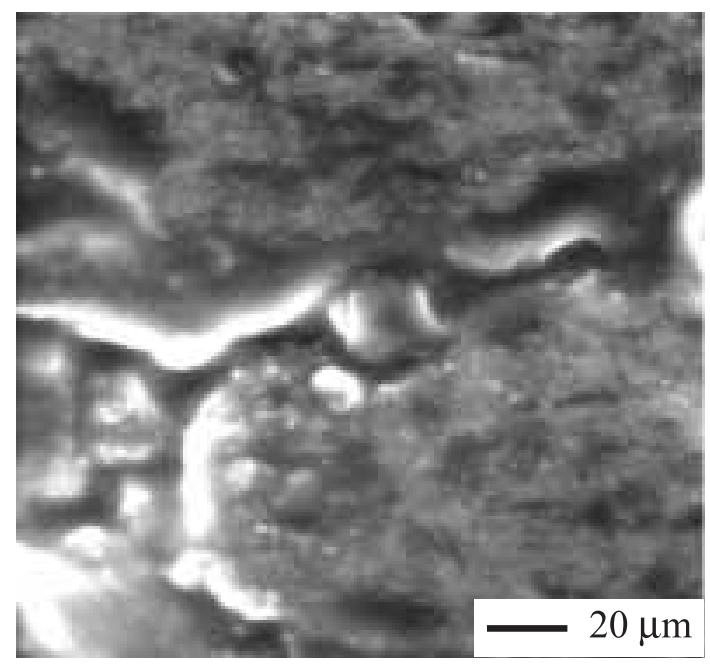

(e)

Figura 2. Micrografia eletrônica de varredura da superfície das amostras de EPDM/PAni-(DBSA) 3 (60/40) processadas por: (a) 5, (b) 10, (c) 20, (d) 30 e (e) $40 \mathrm{~min}$, aumento de 500 vezes. 
DBSA, (ii) reticulação da cadeia da polianilina e (iii) interação com a matriz de EPDM. Todas estas etapas podem acontecer simultaneamente, no entanto, sugere-se uma ordem dos eventos pela observação das curvas de torque. $\mathrm{O}$ primeiro aumento da curva de torque é atribuído, principalmente, à dopagem da PAni. Esta afirmação está baseada em dados de condutividade da amostra em diferentes pontos da curva, verificando-se um patamar após $10 \mathrm{~min}$, independentemente da concentração de $\mathrm{EPDM}^{[13]}$. Em 5 min de processamento a condutividade da blenda é de $10^{-7} \mathrm{~S} . \mathrm{cm}^{-1}$ passando para $10^{-5} \mathrm{~S} . \mathrm{cm}^{-1}$ a partir de $10 \mathrm{~min}$ de processamento. Continuando-se o processamento e aumentando-se o tempo de mistura, verifica-se um segundo acréscimo do torque. Este pode ser atribuído a reticulações da cadeia da polianilina ${ }^{[14,15]}$ ou de EPDM ou a uma interação mais efetiva da PAni com o EPDM. Esta interação já foi anteriormente estudada por ensaios de intumescimento $^{[16]}$. Neste ensaio a amostra é imersa em um solvente para o EPDM (nesse caso utilizou-se ciclohexano). Se o EPDM estiver livre, a fração solúvel (Fs) será igual à concentração deste na blenda. No entanto, verificou-se que Fs era sempre menor, indicando que de alguma forma o EPDM estava interagindo com a PAni ou reticulando. Verifica-se que este solvente, apesar de seletivo para o EPDM, dissolve uma pequena parte de PAni, no entanto, a partir de análises de termogravimetria da amostra solúvel, verifica-se que a quantidade de PAni é apenas $2 \%$. Isto sugere que praticamente a amostra solúvel é de EPDM não reticulado.

As mudanças observadas nas curvas de torque afetam a morfologia da amostra como verificado a seguir. A Figura 2 (a - e) mostra as fotomicrografias de blendas EPDM/PAni-(DBSA) $)_{3}(60 / 40)$ processadas por 5, 10, 20, 30 e 40 min. Em uma ampliação de 500x é possível observar diferenças nítidas na morfologia superficial do material. Em 5 min observa-se uma estrutura bastante compacta que pode ser atribuída, principalmente, à PAni-(DBSA) ${ }_{3}$ que ainda não se misturou com a fase de EPDM. Analisando-se a microscopia de uma amostra processada por 10 min verifica-se um comportamento interessante; a estrutura compacta ainda existe, no entanto, observa-se claramente duas regiões bastante distintas: uma fase lisa de EPDM ainda não misturado à PAni e uma fase de aglomerados de PAniDBSA. Esses aglomerados vão se tornando menores com aumento do tempo de processamento (20 min, Figura 2c), verificando-se o desaparecimento das duas fases distintas e compactas com o aparecimento de uma estrutura "tipo esponja" a 30 e $40 \mathrm{~min}$, Figuras $2 \mathrm{~d}$ e $2 \mathrm{e}$.

\section{Conclusões}

O uso da técnica de microscopia eletrônica de varredura como uma ferramenta para avaliar a morfologia do material, bem como o acompanhamento do processamento, contribuiu para a otimização do processo de obtenção de elastômeros condutores e a interpretação das propriedades finais do material. Todas estas alterações na morfologia podem afetar diretamente a forma de interação da onda incidente com a amostra e, portanto, é possível obter diferentes comportamentos de absorção da radiação eletromagnética do material. Por exemplo, para a amostra processada por 12 min verifica-se uma ressonância na faixa de frequiência de $10,5-11,5 \mathrm{GHz}$ com atenuação média da radiação de $10 \mathrm{~dB}(90 \%$ da radiação incidente) e para a amostra processada por 35 min uma ressonância centrada a $9,5 \mathrm{GHz}$ com atenuação média de $15 \mathrm{~dB}$ (96\% da radiação incidente).

\section{Agradecimentos}

Os autores agradecem à Fapesp (Proc. $n^{\circ}$. 99/03347-2 e 96/09983-0), ao Sargento Duque pelas análises de MEV e a Dermeval Carinhana Jr. pela ajuda na síntese da polianilina.

\section{Referências Bibliográficas}

1. Okabayashi, K.; Goto, F. \& Abe, K. - Synth. Met., 18, p.365 (1987).

2. Jonas, F. \& Morrison, T. - Synth. Met., 85, p.1397 (1997).

3. Angelopoulos, M.; Ray, A.; MacDiarmid, A.G. \& Epstein, A.J. - Synth. Met., 21, p.21 (1987).

4. Angelopoulos, M.; Asturias, G.E.; Erner, S.P.; Ray, A.; Scherr, E.M. \& McDiarmid, A.G., Mol. Cryst. Liq. Cryst. - 160, p.151 (1988).

5. Zoppi, R. \& De Paoli, M.-A. - Polymer.,37, p.1999 (1996).

6. De Paoli, M.-A.; Waltman, R.J.; Diaz, A.F. \& Bargon, J. - J. Chem. Soc. Chem Commun., p.1015 (1984). 
7. De Paoli, M.-A.; Waltman, R.J.; Diaz, A.F. \& Bargon, J - J. Polym. Sci. Polym Chem. Ed.,23, p.1687 (1985).

8. Tassi, E.L. \& De Paoli, M.-A. - J. Chem. Soc., Chem. Commun., p. 155 (1990).

9. Subramaniam, C.K.; Kaiser, A.B.; Gilbert, P.W. \& Wessling, B. - J. Polym. Sci.: Polym. Phys. Ed., 31, p.14245 (1993).

10. Olmedo, L., Houquerbie, P e Jousse, F. em "Handbook of Organic Conductive Molecules and Polymers", Vol.3, H.S. Nalwa (ed), John Wiley, New York, (1997).

11. Freitas, P.S. \& De Paoli, M.-A. - Synth. Met., 102, p.1012 (1999).
12. Titelman, G.I.; Zilberman, M.; Siegmann, A.; Haba, Y. \& Narkis, M. - J. Appl. Polymer Sci, 66, p.2199 (1997).

13. Faez, R.; Martin, I.M.; De Paoli, M.-A. \& Rezende, M.C. - J. Appl. Polym Sci, no prelo.

14. Pereira da Silva, J.E., Faria, D.L.A., Córdoba de Torresi, S.I. \& Temperini, M.L.A.; Macromolecules - 33(8), p.3077 (2000).

15. Pereira da Silva, J.E., Faria, D.L.A., Córdoba de Torresi, S.I. \& Temperini, M.L.A. - Journal of Brazilian Chemical Society, 11 (1), p. 91 (2000).

16. Faez, R. \& De Paoli, M.-A. - Eur. Polym. J., 37, p. 1139 (2001).

Recebido: 16/05/01

Aprovado: 01/10/01 